INPLASY

PROTOCOL

To cite: Cao et al. Mindfulness-

Based Intervention on the

Recovery of Mental Fatigue: A

Systematic Review. Inplasy

protocol 2021120022. doi:

10.37766/inplasy2021.12.0022

Received: 03 December 2021

Published: 03 December 2021

Corresponding author:

Shudian Cao

caoshudian0516@163.com

Author Affiliation:

University Putra Malaysia

Physical Education.

Support: No support.

Review Stage at time of this submission: Data analysis.

Conflicts of interest:

None declared.

\section{Mindfulness-Based Intervention on the Recovery of Mental Fatigue: A Systematic Review}

Cao, S1; Kim, S².

Review question / Objective: Can mindfulness-based intervention recover mental fatigue?

Condition being studied: Mental fatigue: mental fatigue is a psychobiological state caused by a prolonged period of demanding cognitive activity, and it has implicated many aspects of daily life. It results in an acute feeling of tiredness and a decreased cognitive ability. Mindfulness: Mindfulness is rooted in Buddhism, and it is defined as the awareness that emerges from paying attention to objects on purpose and without judging the unfolding of experience. In a successful mindfulness mediation, meditators experience current feelings, thoughts, and bodily sensations clearly and plainly without judging or evaluating them and acting on the sensation.

INPLASY registration number: This protocol was registered with the International Platform of Registered Systematic Review and Meta-Analysis Protocols (INPLASY) on 03 December 2021 and was last updated on 03 December 2021 (registration number INPLASY2021120022).

\section{INTRODUCTION}

Review question / Objective: Can mindfulness-based intervention recover mental fatigue?

Condition being studied: Mental fatigue: mental fatigue is a psychobiological state caused by a prolonged period of demanding cognitive activity, and it has implicated many aspects of daily life. It results in an acute feeling of tiredness and a decreased cognitive ability. Mindfulness: Mindfulness is rooted in Buddhism, and it is defined as the awareness that emerges from paying attention to objects on purpose and without judging the unfolding of experience. In a successful mindfulness 
mediation, meditators experience current feelings, thoughts, and bodily sensations clearly and plainly without judging or evaluating them and acting on the sensation.

\section{METHODS}

Participant or population: Studies with the application of mindfulness-based intervention regardless of population characteristics such as age, gender, and ethnicity.

Intervention: Mindfulness-based intervention.

Comparator: Inclusion criteria: without mindfulness-based intervention and mental fatigue-inducing possibility.

\section{Study designs to be included: RCTs.}

Eligibility criteria: (1) It was ensured that mental fatigue existed. (2) Studies with the application of $\mathrm{MBI}$ regardless of population characteristics such as age, gender, and ethnicity. (3) Randomized controlled trials (RCTs) (4) Intervention: MBI. (5) Comparison: without mindfulness-based intervention and mental fatigue-inducing possibility; two or more groups and singlegroup trials

Information sources: PubMed, Web of Science, EBSCOhost (SPORTDiscus and Psychology and Behavioral Sciences Collection), Scopes, and China National Knowledge Infrastructure (CNKI), Google Scholar.

Main outcome(s): Mindfulness-based intervention is an effective method for the recovery of mental fatigue in psychology and sports performance. It is good for Psychologists and coaches to apply.

Quality assessment / Risk of bias analysis: The quantitative assessment tool 'QualSyst' was used to assess the methodology quality. It contains 14 items (see Table 1). The score depends on the degree to which the specific criteria were met (yes $=2$, partial $=1$, no $=0$ ). Items that do not apply to a particular study design were marked 'NA' and excluded from calculating the summary score. A score of $\geq 75 \%$ indicated strong quality, a score of $55-75 \%$ indicated moderate quality, and a score of $\leq 55 \%$ indicated weak quality.

Strategy of data synthesis: The results (titles and/or abstracts) of studies retrieved using the search strategy and the titles and/or abstracts of studies from other sources will be independently screened by two review authors to identify studies that may meet the above inclusion criteria. The reviewers will review these studies according to the standard of population, intervention, comparison, outcome, and study design. The two review authors will extract data independently, and the differences will be determined and resolved through discussion (discuss with the third author if necessary).

Subgroup analysis: Study design: RCTs; Intervention: Mindfulness-based intervention; Setting: anywhere; Participant characteristics: regardless of population characteristics such as age, gender, and ethnicity.

Sensitivity analysis: None.

Language: No restriction.

Country(ies) involved: China and Malaysia.

Keywords: Mental Fatigue; Mindfulness.

Contributions of each author:

Author 1 - Shudian Cao - The author drafted the manuscript.

Email: caoshudian0516@163.com

Author 2 - Kim Geok Soh - The author provided statistical expertise.

Email: kims@upm.edu.my 